\title{
Submitted: $\quad$ Real-time CEUS-guided biopsy revealing a littoral Accepted: 07.01.2021 \\ Published: cell angioma of the spleen after inconclusive CT and MRI findings
} 16.08.2021

\section{Keywords}

CEUS,

contrast-enhanced ultrasound,

CEUS-guided target biopsy,

littoral cell angioma, spleen

\author{
Markus Herbert Lerchbaumer, Christian E. Althoff, Thomas Fischer
}

Charité - Universitätsmedizin Berlin, Corporate Member of Freie Universität Berlin, Humbold-Universität zu Berlin, and Berlin Institute of Health, Department of Radiology, Berlin, Germany

Correspondence: Markus H. Lerchbaumer, MD, Department of Radiology Interdisciplinary Ultrasound Centre, Charité - Universitätsmedizin Berlin Campus Charité Mitte, Charitéplatz 1, 10117 Berlin, Germany; tel.: +49(0)30 450 657084, fax: +49(0)30 4507557901 , e-mail:markus.lerchbaumer@charite.de

DOI: $10.15557 /$ JoU.2021.0039

\begin{abstract}
The aim of this case report is to present real-time CEUS-guided biopsy for diagnosing rare benign splenic pathologies after inconclusive findings on cross-sectional imaging. We present the case of a 50-year-old male patient who received a contrast-enhanced computed tomography scan of the thorax during the evaluation for lung transplant due to lung fibrosis, with incidental finding of disseminated hypodense splenic lesions. During follow-up imaging, the patient did not tolerate a complete MRI examination, and two acquired pulse sequences did not confirm the final diagnosis. While CT-guided biopsy revealed no results, CEUS-guided target biopsy with repeated contrast injections showed a benign littoral cell angioma of the spleen. The use of real-time CEUS-guided target biopsy during lesion washout may be a useful tool to improve the accuracy of biopsy and accelerate the diagnosis in patients with parenchymal lesions after inconclusive cross-sectional imaging findings which may pose a challenge for CT-guided biopsy.
\end{abstract}

\section{Introduction}

Littoral cell angioma (LCA) is a rare vascular neoplasm of the spleen, composed of littoral cells, i.e. cells that line the splenic red pulp sinuses ${ }^{(1)}$. Previous case studies have suggested an association of LCA with neoplasms of the colon, kidney, pancreas, lung, and ovary (2). Nevertheless, reports on imaging findings are rare, and the final diagnosis is made by histology and immunohistochemistry. In recent years, contrast-enhanced ultrasound (CEUS) has emerged as a reliable tool for guiding real-time biopsy of the liver that has been reported to improve targeting by better detection of lesions without demarcation in B-mode ultrasound ${ }^{(3)}$. The aim of this case report is to present real-time CEUS-guided biopsy in the diagnostic work-up of rare benign splenic pathologies after inconclusive findings on cross-sectional imaging.

\section{Case report}

We present a 50-year-old male patient $(181 \mathrm{~cm}, 97.2 \mathrm{~kg}$, BMI $29.7 \mathrm{~kg} / \mathrm{m}^{2}$ ) listed for lung transplant due to idiopathic pulmonary fibrosis histopathologically confirmed by video-assisted thoracoscopy. While the liver and renal function tests were normal, the patient was in need of oxygen due to stress dyspnoea. A contrast-enhanced computed tomography (ceCT) scan of the chest incidentally revealed multifocal hypodense lesions compared to the surrounding splenic tissue without splenomegaly (with spleen measuring $12.5 \times 4.2 \mathrm{~cm}$ ). During follow-up imaging of splenic findings, the patient did not tolerate a complete magnetic resonance imaging (MRI) examination, and only two pulse sequences could be acquired. The T2-weighted (T2w) images showed multiple hyperintense lesions (Fig. 1A) which were isointense in T1-weighted (T1w) images (Fig. 1B). To 

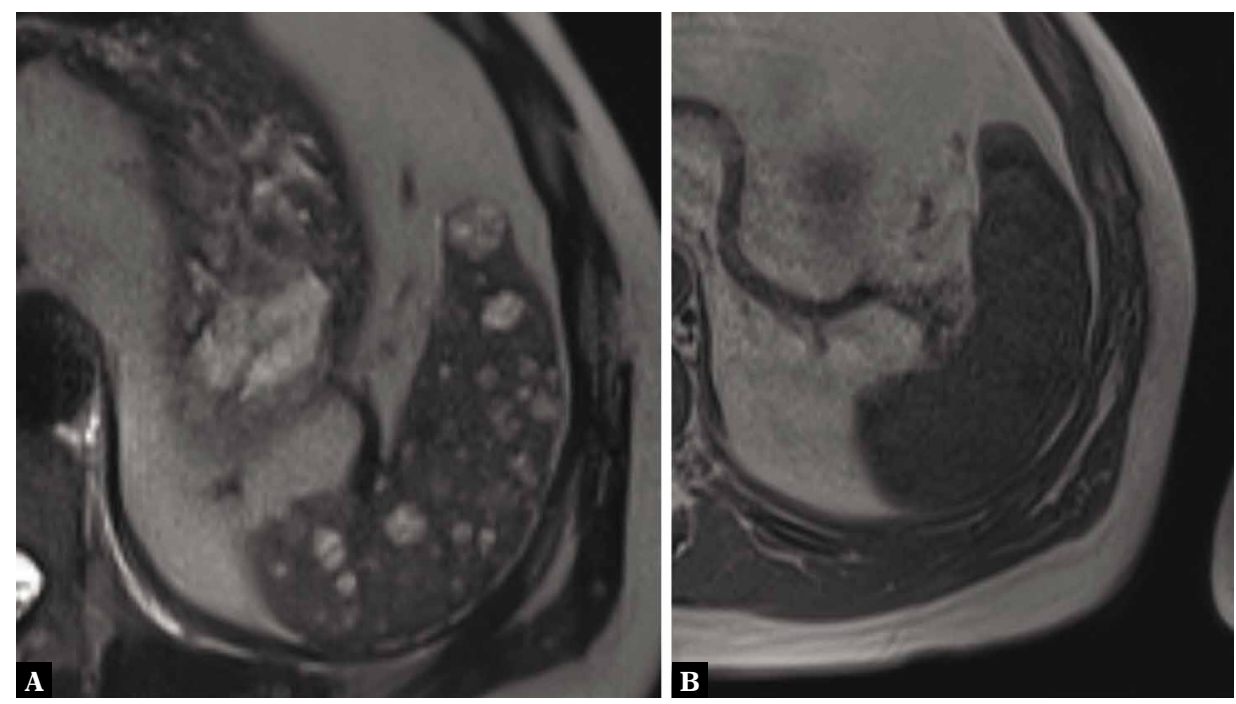

Fig. 1. Magnetic resonance imaging (MRI) of littoral cell angioma. Unenhanced T2w image (a) reveals multiple hyperintense lesions in the spleen (A). The lesions are isointense on T1w imaging (B). Due to dyspnoea, the patient did not tolerate additional sequences or contrast agent injection

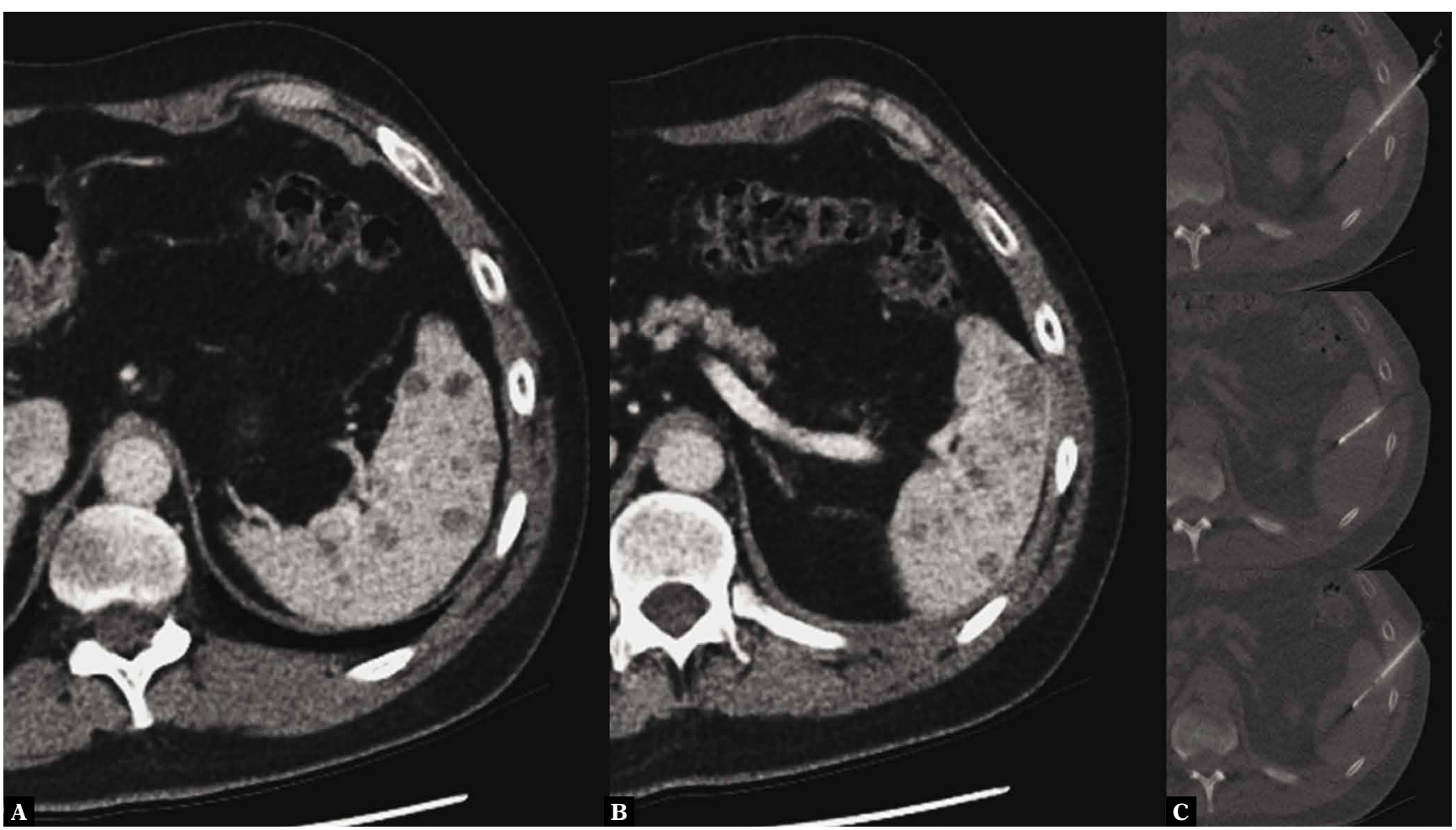

Fig. 2. Contrast-enhanced computed tomography (CT) and biopsy. CT scans acquired 80 sec after injection of 80 ml of iodine-based contrast agent (A, B) show disseminated hypodense lesions throughout the spleen, which is not enlarged. (C) CT-guided biopsy with sampling of three tissue specimens with an 18-gauge needle

exclude malignancy, CT-guided biopsy was performed in the coaxial technique, and three 18-gauge splenic tissue cores were obtained in different needle positions (Fig. 2); however, histopathology revealed no abnormal splenic tissue. Of note, all lesions were isodense on unenhanced CT, and tissue was then sampled after a contrast-enhanced scan for lesion detection and biopsy planning (Fig. 2).
Following this negative CT-guided biopsy, our local interdisciplinary conference opted for US-guided biopsy using CEUS to further work up the patient's splenic lesions. The primary CEUS examination was performed with a convex probe for abdominal use, with a frequency range of 1-6 MHz on a high-end ultrasound system (Aplio i900; Canon Medical Systems Corporation, Tochigi, Japan) with an upto-date CEUS-specific protocol. After a bolus injection of 

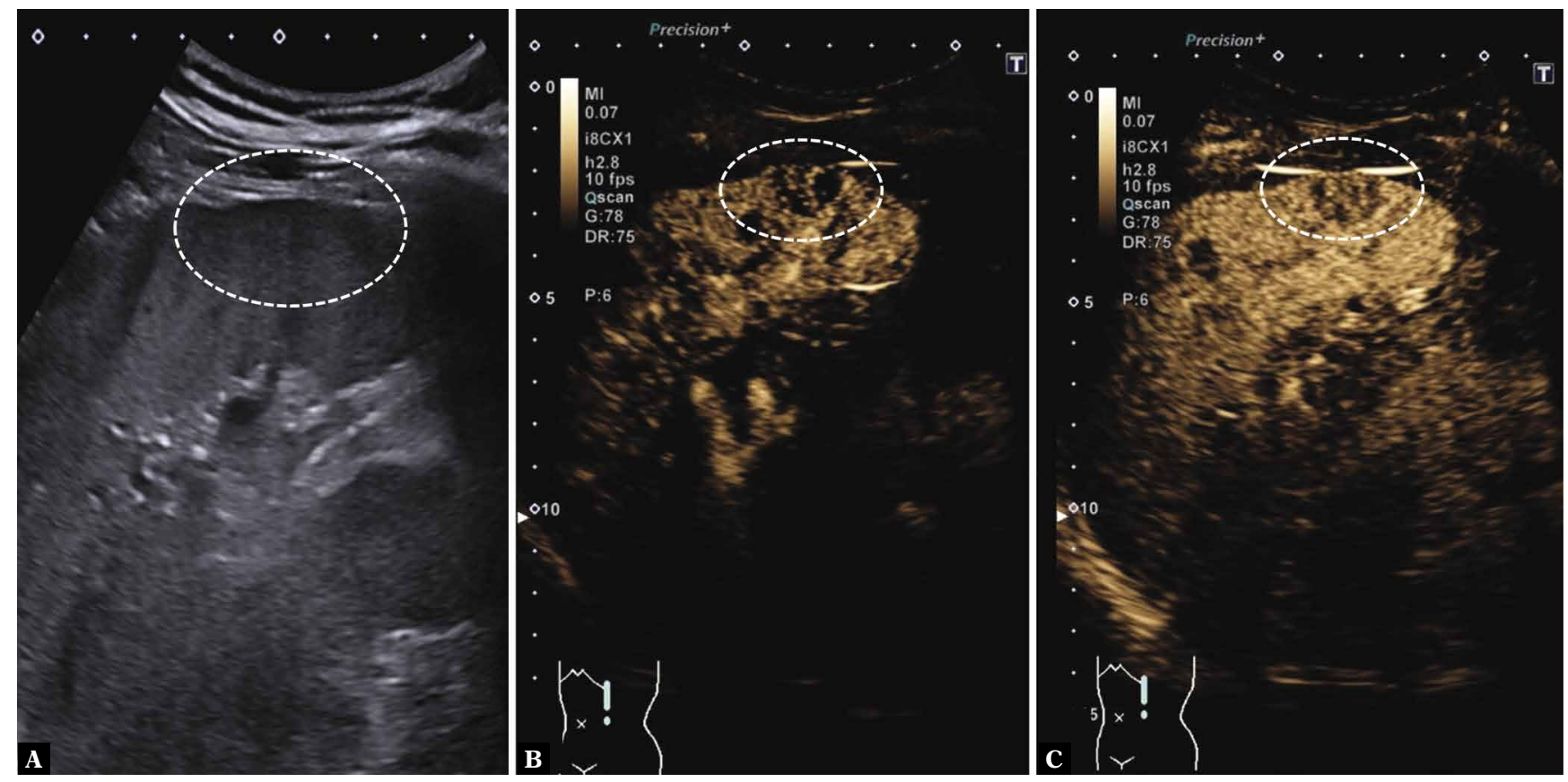

Fig. 3. Contrast-enhanced ultrasound (CEUS) of the spleen. Primary CEUS examination after injection of $1.6 \mathrm{ml} \mathrm{SonoVue^{ \circledR }}$ (Bracco Imaging, Milan, Italy). On B-mode ultrasound, the slightly hyperechogenic target lesion (A) shows no early enhancement (20 sec after injection) and is hypoechogenic in the late phase of the CEUS mode (C)

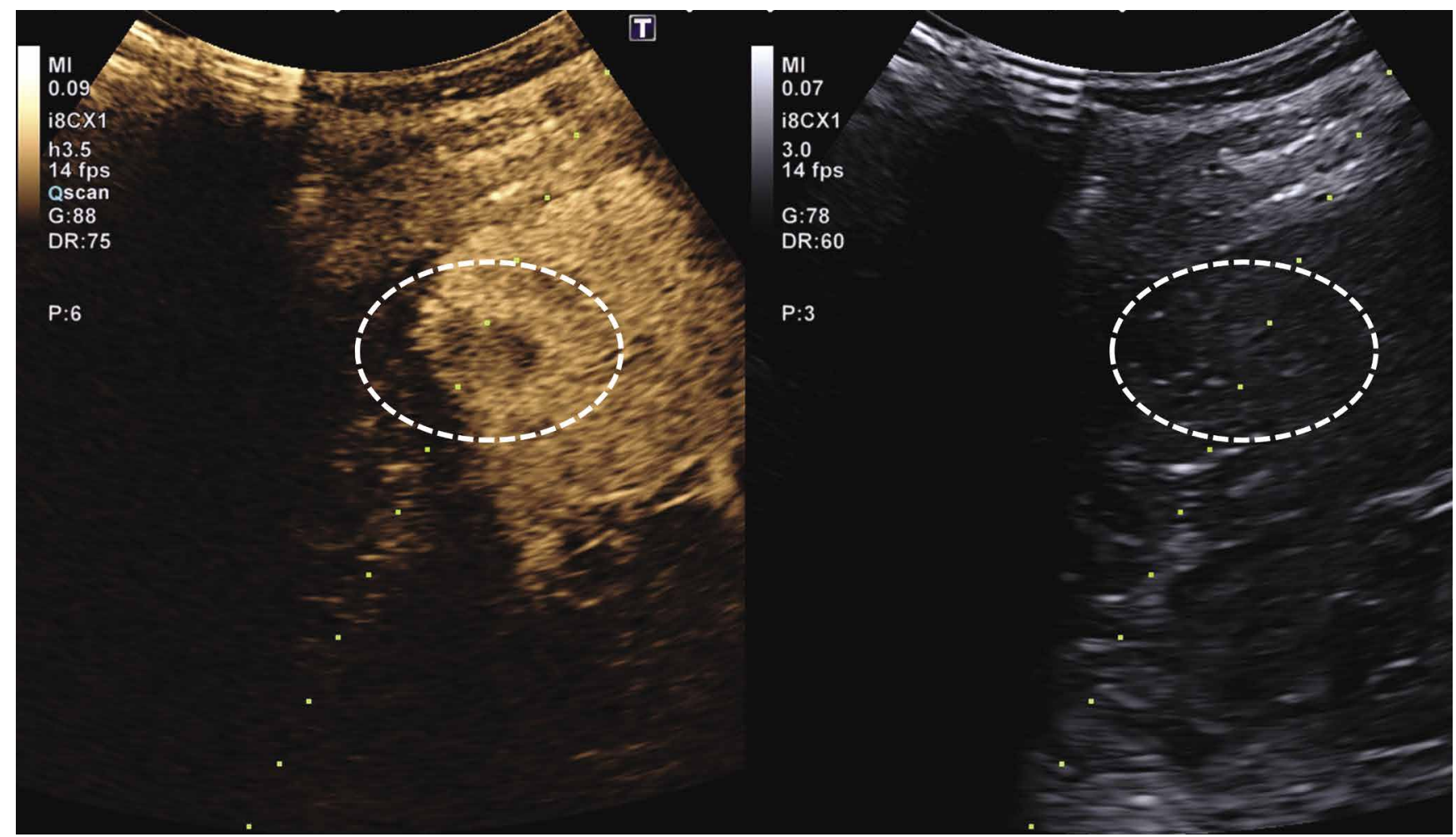

Fig. 4. Real-time CEUS-guided biopsy of the target lesion. After identification of a target lesion (white circle), biopsy is performed with a needle guidance system showing the biopsy marker as a green dashed line with an angle of $65^{\circ}$

$1.6 \mathrm{ml}$ of ultrasound contrast agent (SonoVue ${ }^{\circledR}$, Bracco Imaging, Milan, Italy), CEUS showed subtle hyperechogenic lesions (Fig. 3A) without early enhancement (Fig. 3B) and general hypoenhancement of all disseminated lesions in the late parenchymal phase (Fig. 3C). Intercostal CEUSguided biopsy was performed using a convex probe and needle guidance system (Fig. 4) after a repeated bolus injection of contrast medium $(1.0 \mathrm{ml})$ at 2 -min intervals to 
ensure sufficient enhancement of splenic parenchyma and lesion demarcation.

The histopathological report described B- and T-lymphocytes predominantly with small and chromatin-dense nuclei. Occasionally, meshworks of follicular dendritic cells, CD5, and CD43 were seen, as were T-lymphocytes and CD8 of splenic sinuses. The lymphocytes did not express cyclin D1. Areas of fine-granular-brownish pigment were seen in the cytoplasm, which were positive for CD68 and CD163 as well as for CD31, while CD8 and CD34 were not expressed. There was no increased proliferative activity. Based on these histological findings after CEUS-guided biopsy, littoral cell angioma was diagnosed.

\section{Discussion}

While LCA is often diagnosed after splenectomy, this case nicely illustrates that the diagnosis can be made by the workup of tissue sampled by real-time CEUS-guided biopsy of the spleen with repeated contrast agent injection to ensure adequate tissue contrast and lesion hypoenhancement throughout the examination. Cao et al. showed that real-time CEUSguided biopsy of focal liver lesions during parenchymal or delayed phases improved target lesion conspicuity and thus helped identify active lesion portions ${ }^{(4)}$. This report - as well as our case - thus indicate that CEUS-guided biopsy may spare patients with this benign splenic tumour an unnecessary

\section{References}

1. Falk S, Stutte HJ, Frizzera G: Littoral cell angioma. A novel splenic vascular lesion demonstrating histiocytic differentiation. Am J Surg Pathol 1991; 15: 1023-1033.

2. Bhatt S, Huang J, Dogra V: Littoral cell angioma of the spleen. Am J Roentgenol 2007; 188: 1365-1366.

3. Coran A, Di Maggio A, Rastrelli M, Alberioli E, Attar S, Ortolan P et al.: Core needle biopsy of soft tissue tumors, CEUS vs US guided: a pilot study. J Ultrasound 2015; 18: 335-342. surgical procedure or additional biopsies. In addition, CEUS can provide valuable diagnostic information in the form of real-time data on vascular structures and enhancement patterns of inconclusive splenic lesions ${ }^{(5)}$. While focal splenic lesions are rare in the absence of malignant disease, they are very common in patients diagnosed with lymphoma. In patients with malignant disease, CEUS performed by an experienced examiner can thus make an important contribution to the diagnostic work-up of unclear splenic lesions. Unlike Bhatt et al., who reported isodense lesions compared to surrounding spleen on ceCT due to late filling of the nodules, we saw hypodense lesions on ceCT (Fig. 2) and hypoenhancement on CEUS in our patient with LCA.

LCA is a rare benign entity, and it is often detected incidentally by contrast-enhanced CT or contrast-enhanced MRI. The use of real-time CEUS-guided target biopsy during lesion washout may be a useful tool to improve the accuracy of biopsy and accelerate the diagnosis in patients with parenchymal lesions of the liver, spleen or kidney, and inconclusive cross-sectional imaging findings which may pose a challenge for CT-guided biopsy.

\section{Conflict of interest}

Authors do not report any financial or personal connections with other persons or organizations which might negatively affect the contents of this publication and/or claim authorship rights to this publication.
4. Cao X, Liu Z, Zhou X, Geng C, Chang Q, Zhu L et al.: Usefulness of real-time contrast-enhanced ultrasound guided coaxial needle biopsy for focal liver lesions. Abdom Radiol 2019; 44: 310-317.

5. Lerchbaumer MH, Kleemann T, Jung EM, Nagel S, Hamm B, Fischer T: Vascular pattern and diagnostic accuracy of contrast-enhanced ultrasound (CEUS) in spleen alterations. Clin Hemorheol Microcirc 2020; 75: 177-188. 